\title{
Develop of CFO in High Mobility Environments for OFDM-DMWT Based Mobile Wireless Applications
}

\author{
Dr. Mohammed Aboud Kadhim \\ Middle Technical University, Institute of Technology Baghdad, Iraq
}

\begin{abstract}
An "Orthogonal Frequency division Multiplexing (OFDM)" systems are sensitive to "service Frequency Offset (CFO)" mistakes. OFDM- "Discrete Multi-wavelet remodel (DMWT)" based totally cellular wireless with a sender, a provider Frequency Offset errors turns into similarly tough for cellular applications in view that power postpone outline of the channel may trade quick because of the sporadic start and dying of the channel paths. We discover analytical expressions for channel estimation mistakes inside the existence of CFO mistakes and excessive mobility. We display that the sensitivity of the channel estimator be capable of still be exploited to increase CFO in high mobility environments. A comparative have a look at amongst extraordinary techniques of service frequency offset estimation in OFDM systems is offered. We keep in mind strategies in frequency area are considered, namely; training image approach and Pilot tone technique. Mean square blunders (MSE) is the evaluation criteria used within the look at. Simulation consequences display that the CFO estimation strategies the schooling image technique outperforms the pilot tone approach with the aid of about $2 \mathrm{~dB}$. The contrast results are considered at MSE of 10-4 and normalized CFO of 0.2 .
\end{abstract}

Keywords- CFO, ICI, BER, SNR, OFDM, DMWT, MSE, Frequency domain.

DOI: $10.7176 / \mathrm{NCS} / 10-04$

Publication date:July $31^{\text {st }} 2019$

\section{I.INTRODUCTION}

Telecommunication market place has advanced quickly in the ultimate many years, generating a want for new techniques that can adapt excessive data charges. Numerous virtual communications services has been deployed with constantly increasing information fees comparable digital radio, virtual tv, and cellular net. One of the essential topics in current broadband structures is the usage of OFDM. OFDM is a modulation scheme appropriate for frequency selective channels and for giving high velocity information transmission, which makes it one of the promising solutions for the subsequent generation wireless communications. OFDM mitigates the impact of multipath channel by using basically partitioning the supply variety into numerous narrow sub-bands which might be transmitted within the intervening time. In OFDM scheme, the supply bit-movement to be transmitted over the air connection is a component into $\mathrm{N}$ parallel streams, which are later going to be modulated utilizing $\mathrm{N}$ sub- carriers. In light of utilizing sub-carriers -transporters, the symbol duration gets to be $\mathrm{N}$ times bigger. This declines and even thoroughly deflects the impact of entomb "Inter symbol Interference (ISI)" in multipath channels, and therewith lessens the equalization complication. All things considered, there is a requirement for further developments of OFDM schemes as far as complication diminishment and adjustment, it takes after reconfigurable arrangements are important to accomplish the client necessities. This is required in light of the fact that the end clients need lightweight, smaller size and power qualified devices other than the high bit rate faculty[1,2]. OFDM is very sensible to time and frequency synchronization. The synchronization difficult contains of two main parts: carrier frequency offset (CFO) and symbol time offset (STO) [3]. This OFDM synchronization at the receiver is one significant step that necessity be achieved. Synchronization of an OFDM signal needs finding the symbol timing and carrier frequency offset. Finding the symbol timing for OFDM means finding an estimate of when the symbol starts. There are two chief reasons of CFO. The first is a frequency mismatch between the local oscillators at the transmitter and receiver, which outcomes in residual $\mathrm{CFO}$ at the receiver after the down-conversion method. The second reason is the Doppler shift, which is a result of the comparative motion between the transmitter and receiver current in mobile surroundings [4, 5]. The normalized CFO can be separated into two parts; integral CFO (IFO) and fractional. CFO (FFO). IFO produces a cyclic shift by in receiver side to consistent subcarrier it does not destroy orthogonally among the subcarrier frequency components. However, FFO destroys the orthogonally between the sub-carriers. There are numerous difficulties related with the carrier signal like there might be some phase distortions due to error of carrier signal generation at the transmitter and receiver ends. Another general difficult inherited in the carrier signal is the CFO which is caused by the Doppler frequency shift with the varying velocity when passing through the medium. This CFO too happens due to differences between the oscillators at the transmitter and receiver side affected by the system noise temperature at both correspondingly [6, 7]. "Discrete Multi-wavelet Transform (DMWT)" offers an equivalent to the well-established OFDM as a proficient multicarrier modulation method. It has strong benefit of being generic transmission system whose actual features can be extensively modified to achieve numerous necessities and restraints of progressive communication schemes. In the previous decades wavelets 
have been approvingly applied in signal and image processing fields but they just lately fascinated attention of the telecommunication group. So, various study questions continue to be addressed before DMWT be able to be used in practice. One of the main handle contain the performance of DMWT transceivers under numerous synchronization errors. In this work we examine the interference in DMWT transmission affected by the carrier frequency offset and time synchronization errors. Using standard DMWT, the sensitivity of transceivers to these errors is estimated within simulation studies and their performances are compared and compared to OFDM. To extenuate the DMWT, s susceptibility to time synchronization errors, a technique of synchronization is suggested [8]. In this paper to mitigate the effects of CFO in OFDM-DMWT scheme have shown a benefit of improving system performance in terms of "bit error rate (BER)" against "signal-to-noise ratio (SNR)". To steady the attained results, also compares the performances of the scheme developed in this paper with the extensively known and used OFDM-DMWT scheme with self-cancellation CFO estimator the rest of this paper is organized as follows. Frequency-domain estimation methods are presented in section II. Simulation results are presented in section III. . Finally, conclusions are drawn in Section IV

\section{II.Frequency-Domain Estimation Methods for CFO}

Frequency Domain (FD) CFO Estimation techniques [9] are applied under supposition that perfect time synchronization is attained. Additionally, the FD methods are based on transmitting two identical symbols or pilot tone (pilot insertion) [10]. In CFO estimation using frequency domain, this technique involves the comparison of the phase of the each sub carrier to successive symbol, the phase shift in symbol due to the carrier frequency offset [11]. Two different estimation modes for CFO estimation in pilot based estimation method is used which are acquisition and tracking mode. In the acquisition mode large range of CFO estimation[12] is done and in tracking mode only the fine CFO is estimated. Initially we assume that acquisition estimation is already performed and hence fine CFO estimation is performed in this paper. All simulation results show mean square error (MSE) with respect to different signal to noise ratio (SNR) in $\mathrm{dB}$ and compared for training sequence with ratio of OFDM symbol to repetitive sequence length with respect to different CFO value.

\section{III.SYSTEM MODEL}

Regard as OFDM-DMWT Transmitter and Receiver are displayed in Figure.1,

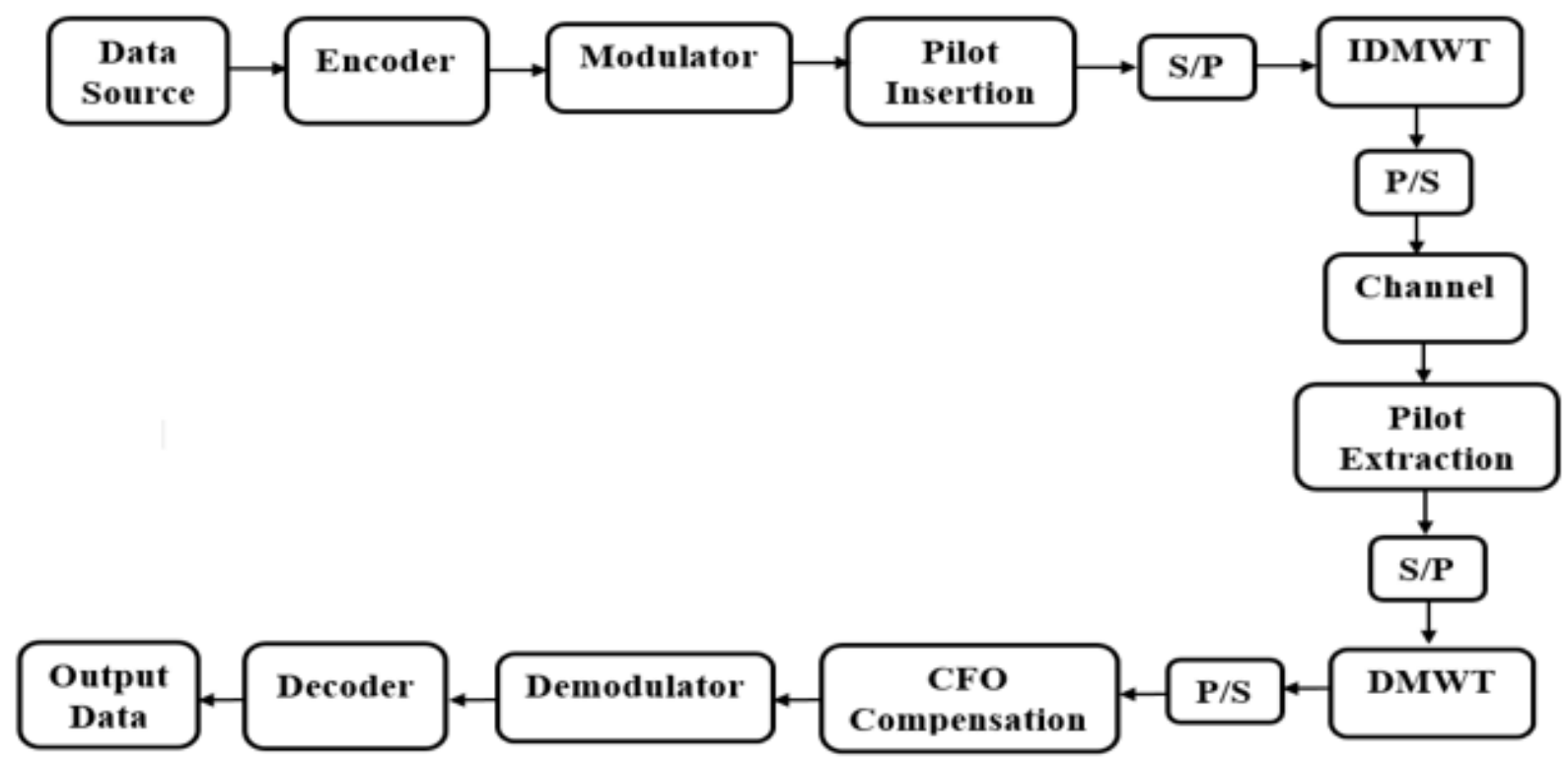

\section{Figure 1: Block diagram of OFDM-DMWT Transmitter and Receiver}

The data source produces symbol which be related to M-PSK, 16-QAM, or 64-QAM and are supposed to be individual and equiprobable. The symbolic sequence is serial to parallel converted into block of $\mathrm{N}$ symbols.

$$
\psi_{l, k}(t)=\left\{\begin{array}{cl}
e^{j 2 \pi f_{k}(t-l T)} & 0<t<T \\
0 & \text { elsewhere }
\end{array}\right.
$$

OFDM signal in Continuous time domain. Where $n_{k}$ are the data, $N$ is the number of sub-carriers, and $T$ is the data duration. The DMWT in OFDM with, the transmitted signal can be expressed as 


$$
x_{l}(t)=\frac{1}{\sqrt{2 \mathrm{D}_{\mathrm{r}}}} \sum_{\mathrm{d}=0}^{2^{D_{-1}}} \sum_{i=0}^{\mathrm{r}} \mathrm{n}_{\mathrm{d}, \mathrm{i}} \Phi_{\mathrm{d}, \mathrm{i}}(\mathrm{t}), \quad 0 \ll \mathrm{t} \ll \mathrm{T},
$$

Effect of channel on received baseband symbol seeing the effect of channel noise at receiver.

$$
Y_{l}(k)=H_{l}(k) X_{l}(k)+N_{l}(k)
$$

$X_{l}(k), Y_{l}(k), H_{l}(k)$ and $N_{l}(k)$ denote $k^{\text {th }}$ subcarrier frequency component of $l^{\text {th }}$ transmitted symbol, channel frequency response and noise in frequency domain.

$$
y_{l}(n)=\operatorname{IDMWT}\left[Y_{l}(k)\right]
$$

Received signal is multiplied by a carrier signal for the purpose of frequency up-conversion. At the receiver section, there is a need of frequency down conversion. But since these signals are generated by local oscillators which are seldom synchronized at both end transmitter and receiver. It may cause carrier frequency offset, which degrades signal quality and can affect orthogonally of subcarriers. Effect of carrier frequency offset (CFO) on received can be modeled as a time variant phase shift, the received OFDM signal can be written as

$$
y_{l}(n)=\frac{1}{N} \sum_{k=0}^{N-1} H_{l} X_{l}(k) e^{j 2 \pi \frac{(k+\varepsilon)}{N}}+\operatorname{IDMWT}\left[N_{l}(k)\right]
$$

CFO arises due to frequency offset .let $f_{c t r}$ and $f_{\text {cre }}$ denote carrier frquency in transmitter and rceiver and difference denoted by

$$
f_{\text {offset }}=f_{c t r}-f_{c t r}
$$

Where CFO $\epsilon=\frac{f_{\text {off set }}}{\Delta f}, \Delta f=$ subcarrier spacing, Doppler frequency is estimated by $f_{\text {ctr }}=\frac{v \cdot f_{c}}{C}$, where $\mathrm{C}$ denote speed of light

Taking DMWT of $y_{l}(n)$ in frequency domain received signal will be

$$
Y_{l}(k)=\frac{\sin \pi \varepsilon}{N \sin \left(\frac{\pi \varepsilon}{N}\right)} e^{j 2 \pi \varepsilon(N-1)} H_{l}(k) X_{l}(k)+U_{l}(k)+N_{l}(k)
$$

Where

$$
U_{l}(k)=e^{j 2 \pi \varepsilon(N-1)} \sum_{s=0, s \# k}^{N-1} \frac{\sin [\pi(s-k+\varepsilon)]}{N \sin \left[\frac{\pi(s-k+\varepsilon)}{N}\right]} H(S) X_{l}(s) e^{j \pi(s-k)\left(\frac{N-1}{N}\right)}
$$

Where $\frac{\sin [\pi \varepsilon]}{N \sin \left[\frac{\pi(\pi \varepsilon)}{N}\right]}$ denoted the amplitude and phase distortion of received signal Moose [9] gives the MLSE (Maximum likelihood sequence estimator) for carrier frequency offset estimation. The limit of acquisition range for the carrier frequency is. Classen [8] proposed a technique in which pilot can be inserted and transmit in OFDM symbol for CFO estimation. Pilots can be inserted in frequency domain and transmit in each OFDM symbols for CFO tracking. Pilot has been designed to minimize MSE, improve channel capacity, channel estimation and minimizing symbol error rate. One OFDM symbol is transmitted instead of two symbols, then, the signals are transformed into via DMWT. After estimating CFO from pilot tones in the frequency domain, the signal is compensated with the estimated CFO in the time domain. In this process, two different estimation modes for CFO estimation are implemented: acquisition and tracking modes. Acquisition mode benefited by aid of pilots subcarriers, which are known at receiver. CFO acquisition can be divide into two steps, the integer part 
and fractional part. In the acquisition mode, a large range of CFO including an integer CFO is estimated. In the tracking mode, only fine CFO is estimation.

$$
\varepsilon_{a c q}=\frac{1}{2 \pi} \max _{\varepsilon}\left[\left|Y_{l}(p(k), \varepsilon), X_{l} p(k)\right|\right]
$$

Where $\mathrm{L}$ is number of subcarriers, $\mathrm{p}(\mathrm{k})$ is location of $k^{\text {th }}$ pilot tone $X_{l} p(k)$ is the pilot tone located at $\mathrm{p}(\mathrm{k})$ in frequency domain at $1^{\text {th }}$ symbol period in transmitted signal.

\section{Simulation and Results}

In this part the performance of the High Mobility the user-channel vehicular A profile with $60 \mathrm{~km} \mathrm{~h}$ is addressed and the High Mobility the user-channel vehicular B profile with 120kmh is presented [13]. An OFDM structure with 64-QAM modulation, 256 subcarriers. Proposed structure contain pilots at four spaces separated. All pilot signs are supported by some degree or signal power utilized is ideal, so it doesn't cost high as far as power. We contrast MSE execution and Moose, Moose[14] approach requires a unique prelude period and estimation range of CFO is. This approach depends on MLSE (Maximum Likelihood Sequence Estimator). Classen present a structure uses trial and error technique to find CFO which is computationally complex [15]. Proposed method utilizes a supported pilot subcarrier touches base at recipient. Beneficiary initially gained flag and concentrate pilot subcarrier. This system has computationally less perplexing and performs well contrast with others. Indemnified CFO signal is appeared by BER charts. In the wake of evaluating CFO, it repays by pay circuit and we find that it gives perfect BER execution

\section{A. Mean Square Error Performance}

Channels vehicular A and B are evaluated at receiver and it is redressed by equalization. OFDM system are reenacted considering structure with 256 point DMWT and pilot dividing is four. CP comprises of back some portion of OFDM symbol, which is made out of tests. Convolution code is appropriate for synchronization to give ideal execution, CFO estimation range can be expanded however it might debases the execution of MSE. $\mathrm{CFO}=0.2$ and 0.4 is determined for and at various estimations of SNR as appeared in Figures 2, 3, 4 and 5.

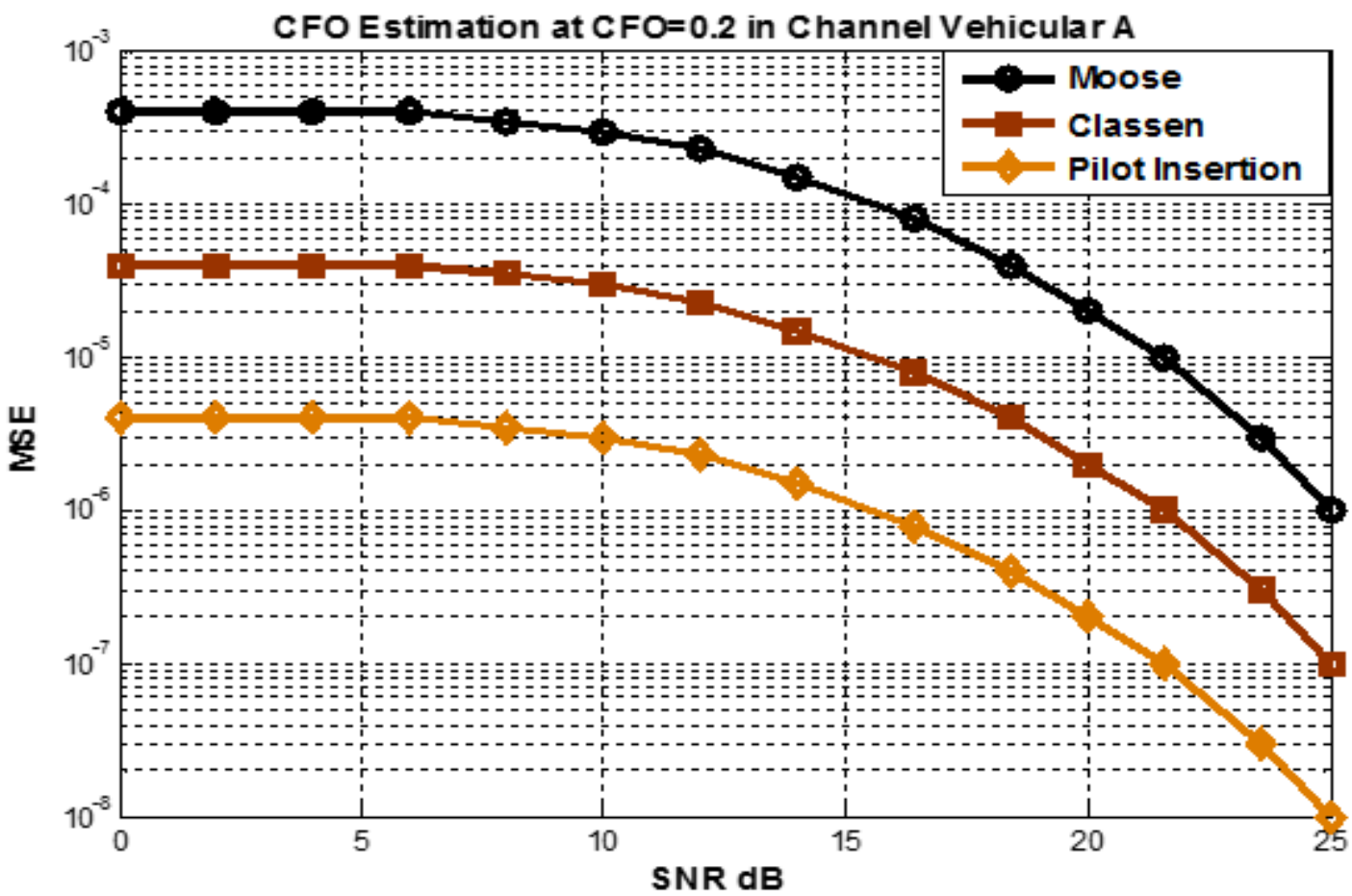

Figure 2: $\mathrm{CFO}$ Estimation at $\mathrm{CFO}=0.2$ in Channel Vehicular $\mathrm{A}$ 


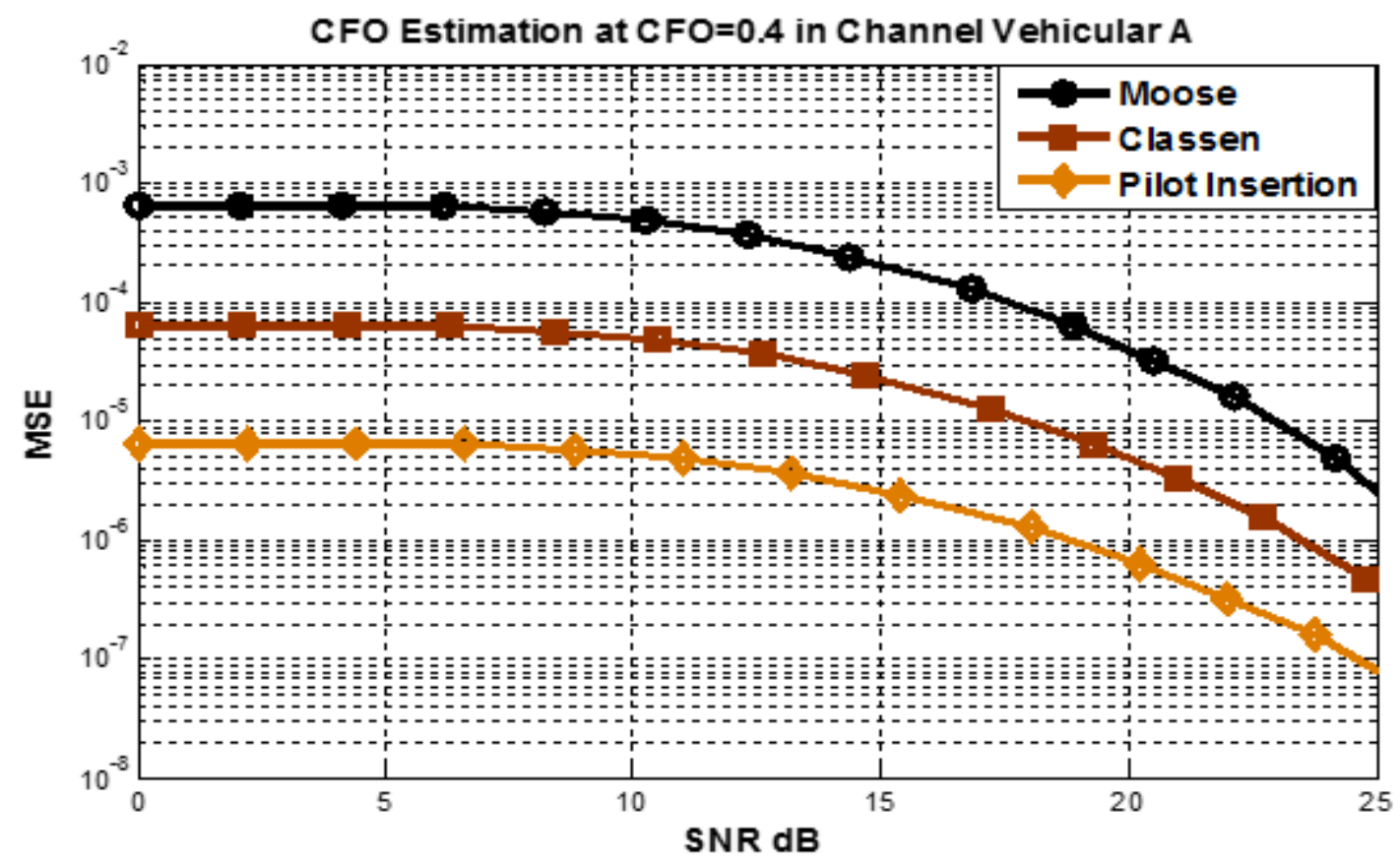

Figure 3: $\mathrm{CFO}$ Estimation at $\mathrm{CFO}=0.4$ in Channel Vehicular $\mathrm{A}$

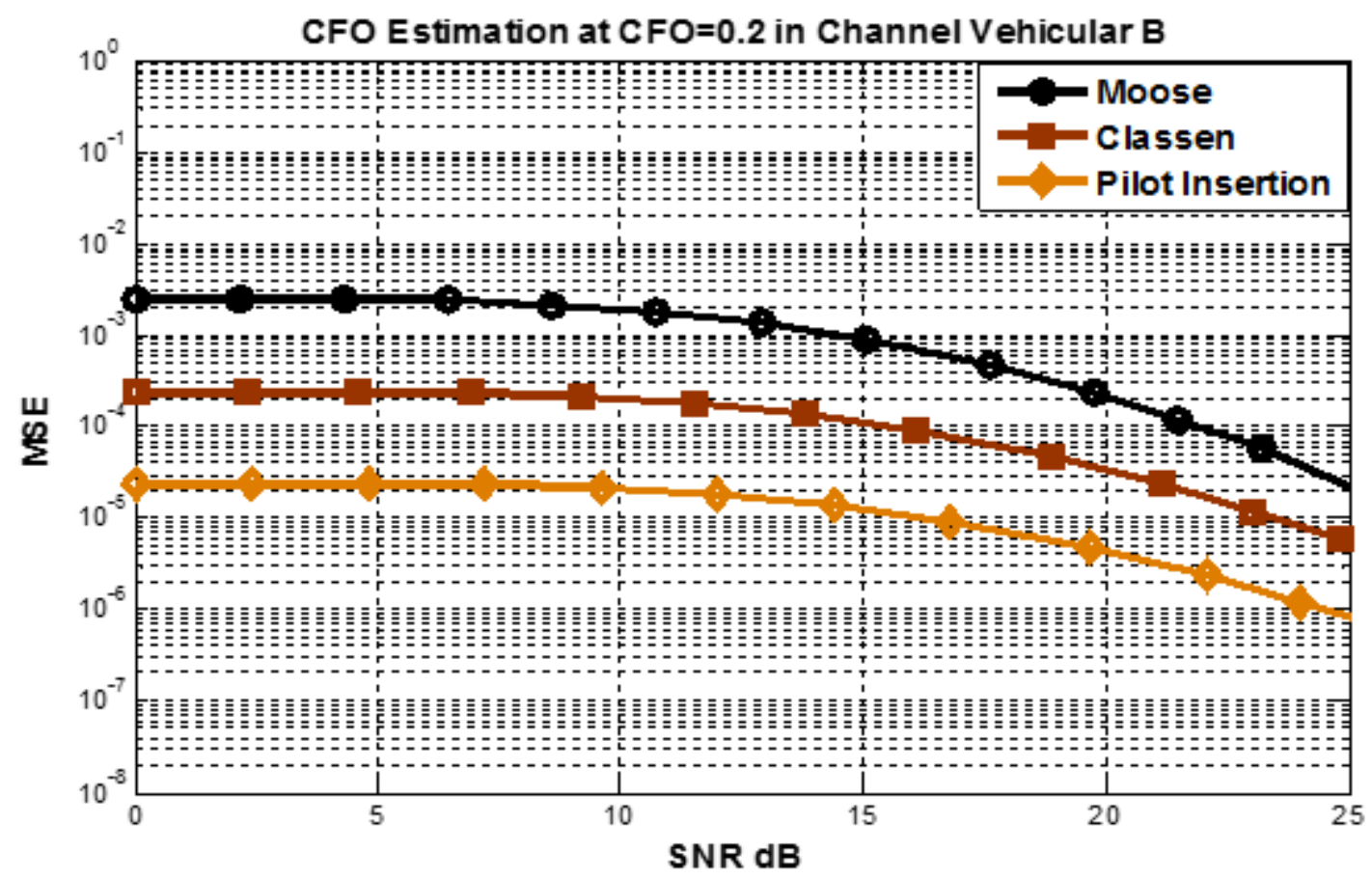

Figure 4: $\mathrm{CFO}$ Estimation at $\mathrm{CFO}=0.2$ in Channel Vehicular $\mathrm{B}$ 


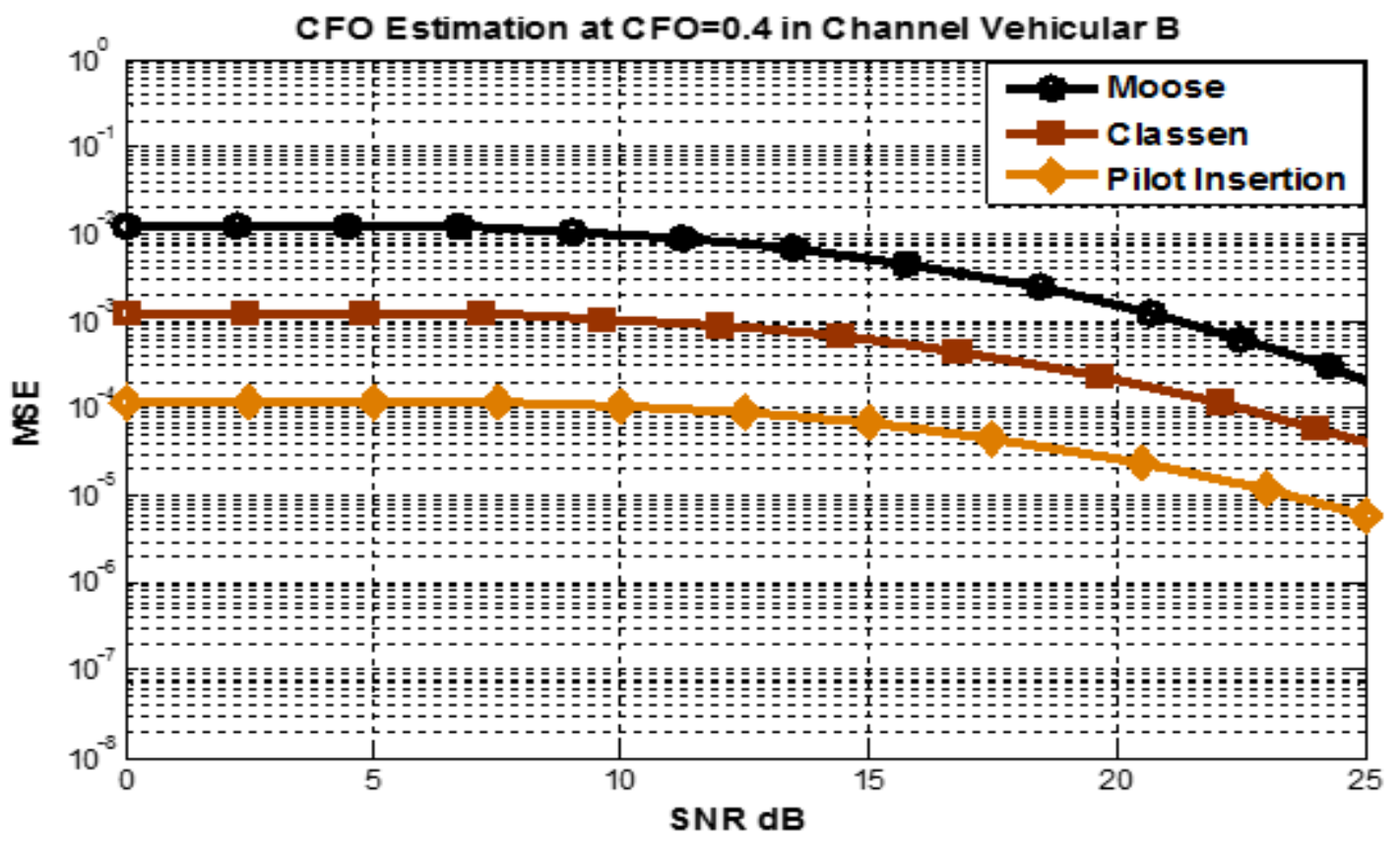

Figure 5: $\mathrm{CFO}$ Estimation at $\mathrm{CFO}=0.4$ in Channel Vehicular $\mathrm{B}$

Estimation execution depends on MSE versus SNR in dB. MSE. Moose approach in view of two back to back symbols amid preface and technique neglects to evaluate exact recurrence balance. It is pertinent in the span of prelude period when image can't be transmitted. Classen technique removed pilots at frequency domain, it measure CFO precisely yet comes up short when CFO values goes higher. As CFO expands, MSE execution may decrease as appeared in Figures 3 and 5. On the off chance that surpass to estimation go, it won't conceivable to measure CFO precisely. Figure 5 demonstrates that Classen approach is falling behind different techniques implies if CFO esteem expands, reiteration of symbol won't perform great estimation. For this situation pilot based proposed idea is great regarding MSE.

\section{B. BER Performance}

In this part, the MATLAB simulation is achieved to assess BER execution. The simulation parameters are as per the following: there are 256 subcarriers including 32 pilots, which are four separating separated with each other, Convolution coding is done at rate of $1 / 2$. At Decoder side hard choice interpreting is performed. Convolution code is reasonable for synchronization to give ideal execution. Tweak conspire utilized 64-QAM; in Channel Vehicular A and B, all SNR qualities are in dB. BER performance shown in Figures 6,7,8 and 9, when CFO is inserted in OFDM-DMWT. 


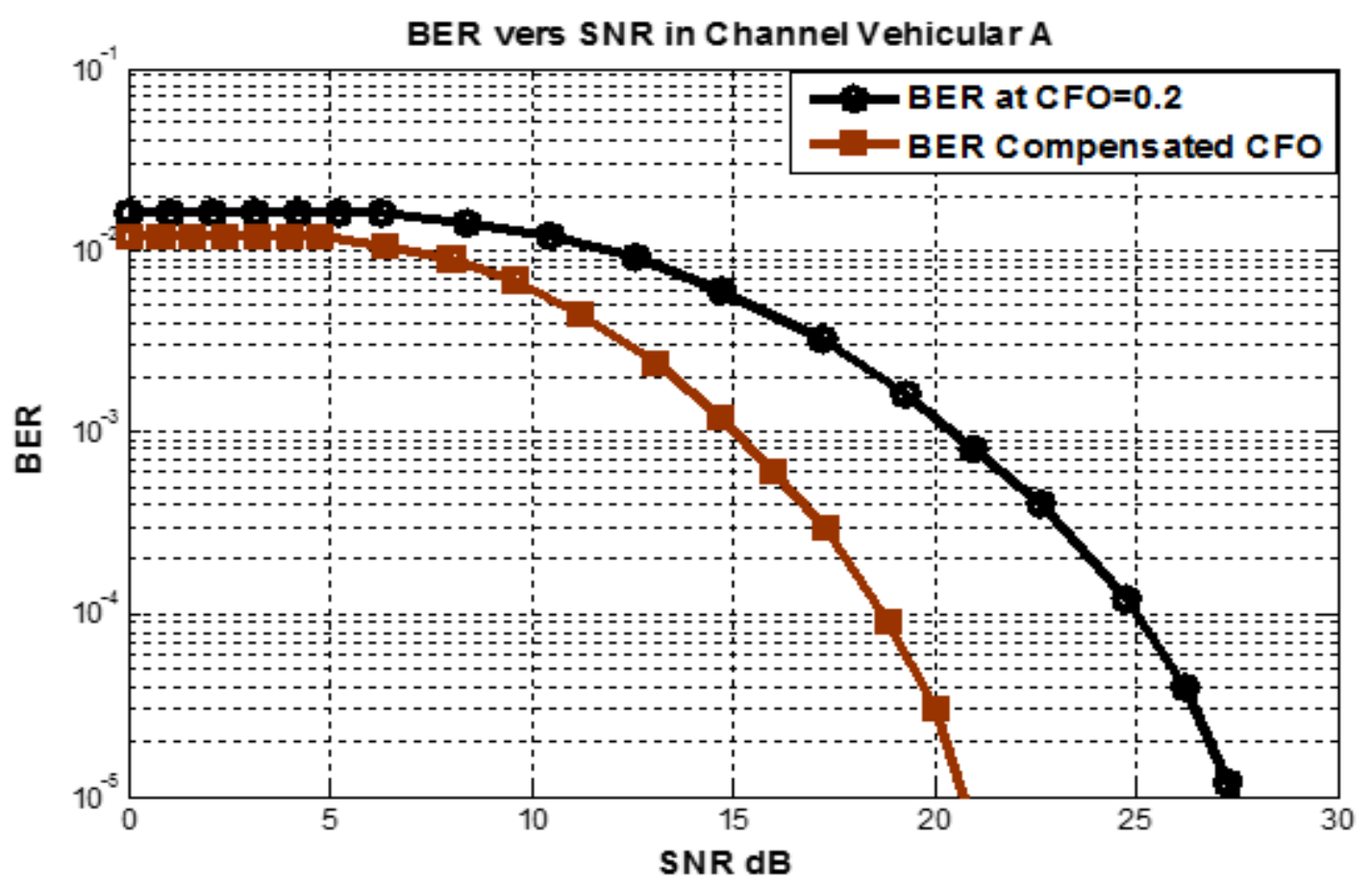

Figure 6: $\mathrm{BER}$ vers $\mathrm{SNR}$ when $\mathrm{CFO}=0.2$ and Compensated $\mathrm{CFO}$ in Channel Vehicular $\mathrm{A}$

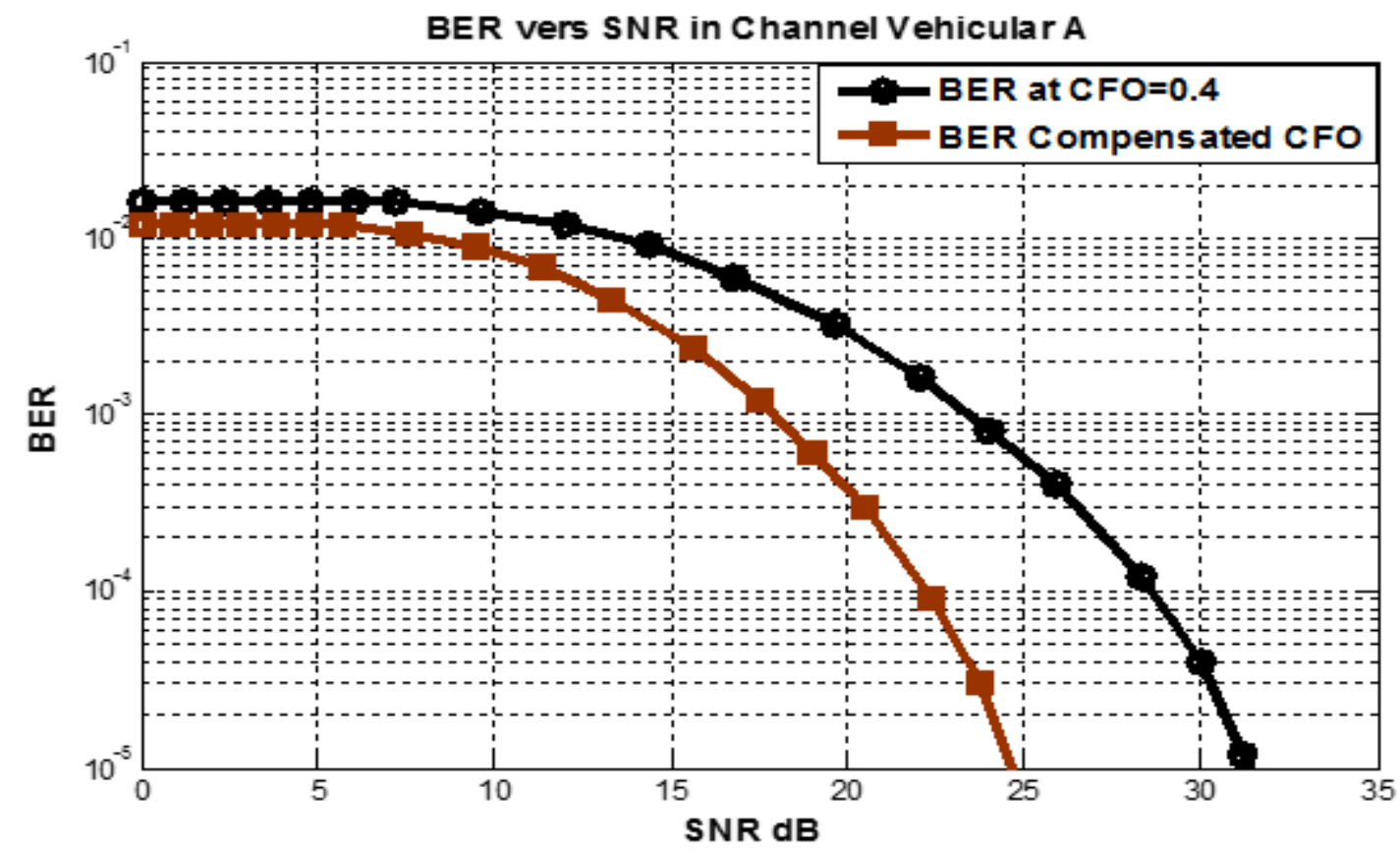

Figure 7: $\mathrm{BER}$ vers $\mathrm{SNR}$ when $\mathrm{CFO}=0.4$ and Compensated $\mathrm{CFO}$ in Channel Vehicular $\mathrm{A}$ 


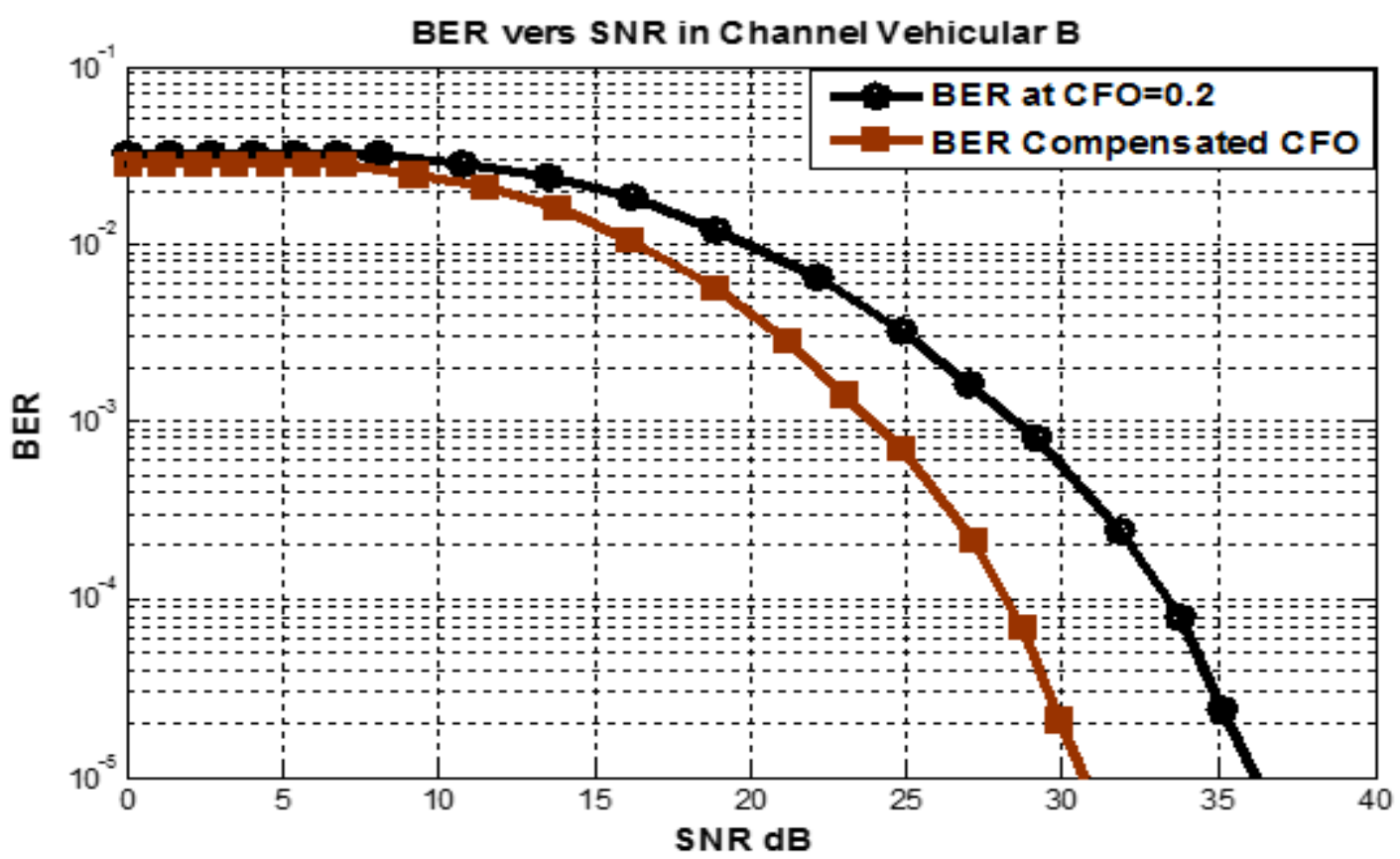

Figure 8: $\mathrm{BER}$ vers $\mathrm{SNR}$ when $\mathrm{CFO}=0.2$ and Compensated $\mathrm{CFO}$ in Channel Vehicular $\mathrm{B}$

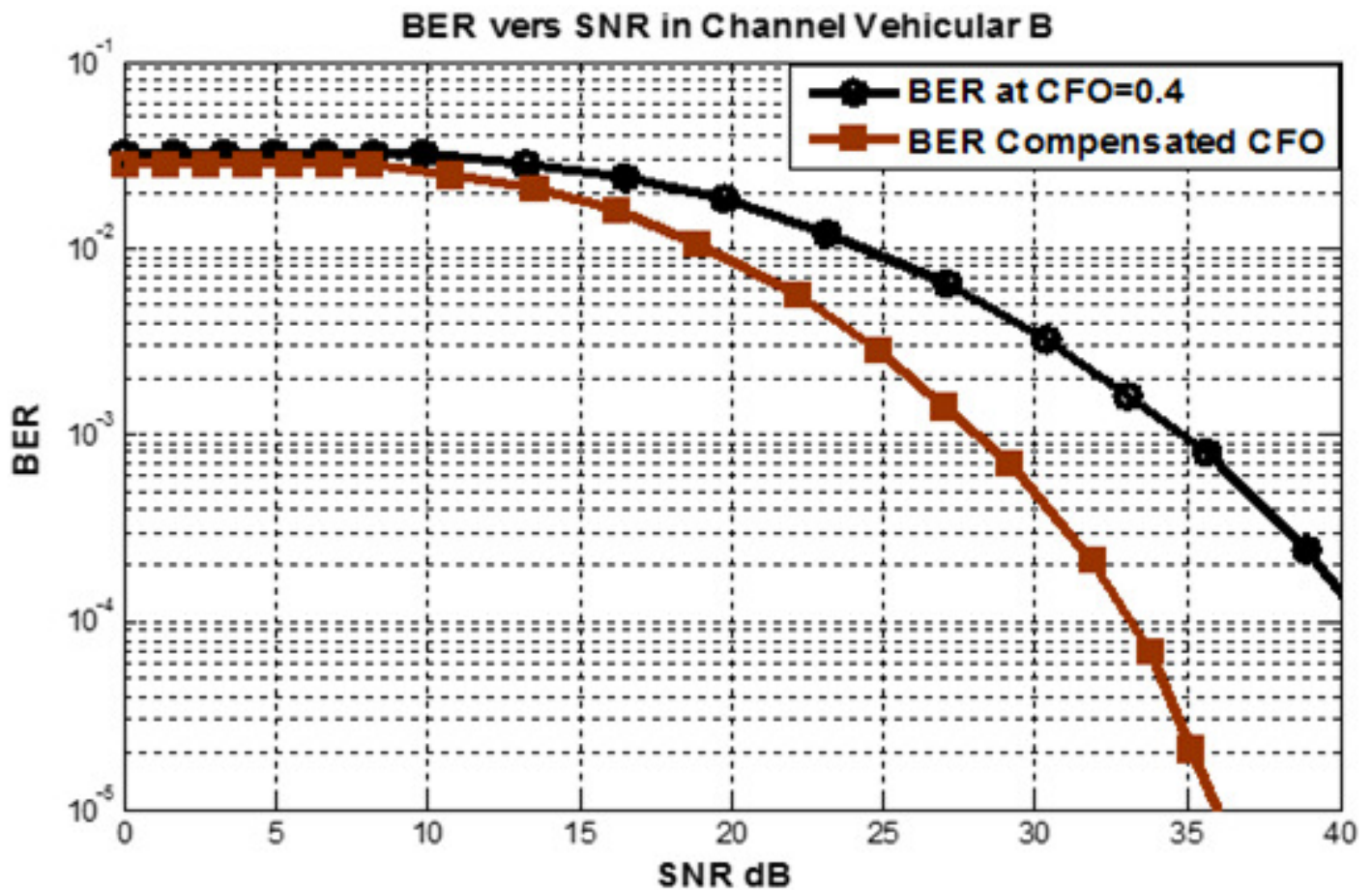

Figure 9: $\mathrm{BER}$ vers $\mathrm{SNR}$ when $\mathrm{CFO}=0.4$ and Compensated $\mathrm{CFO}$ in Channel Vehicular $\mathrm{B}$

Standardized Carrier frequency offset esteem for which BER computed is estimation execution depends on MSE versus SNR in dB. MSE will be computed .Moose technique in view of two back to back signals amid prelude and approach neglects to assess precise frequency offset. It is pertinent in the span of introduction period when symbol can't be transmitted. Classen technique removed pilots at frequency domain, it assess CFO precisely however fizzles when CFO values goes higher. BER execution appeared in fig 6, when CFO is embedded in OFDM system, BER execution get decrease. As CFO builds BER get increments as appeared in Figures.6, 7, 8 and 9. 


\section{V.Conclusion}

Three estimators' method for CFO estimation in Channels Vehicular A and B conditions were proposed in this paper. CFO difficult is considered and a technique has been presented based on insert pilot alignment to estimate CFO. Offered procedure work on frequency domain and estimated CFO is compensated. It too considered by low computational complexity. The simulation result show the good performance of our proposed insert pilot assisted system. MSE calculate performance of suggested system based, Moose and Classen method. Benefit of this technique is that there is no necessity of repeating sequence to estimate CFO. BER result demonstrations that compensated OFDM symbol mends BER performance at diverse values of SNR (dB). In this work we researched carrier frequency offset issue and frequency synchronization in frequency domain for OFDM-DMWT structure in view of pilot helped plot utilizing inert pilot alignment .

\section{References}

[1] A. M. C. O. Shin, H. T. Kung, and V. Tarokh,, "Design of an OFDM cooperative space-time diversity system," IEEE Trans. Veh. Technol., vol. 56, pp. 2203-2215, 2007, 2007.

[2] Prasad R, " OFDM for Wireless Communications Systems. ," London: Artech House, Inc; , 2004.

[3] F. Xiong, "'Digital Modulation Techniques", Second Edition, Artech House, Boston, London , pp. 745797.," 2006.

[4] W. Aziz, Ahmed, E., Abbas, G., Saleem, S. and Islam, Q., , "“Performance Analysis ofcarrier Frequency Offset (CFO) in OFDM using MATLAB”, Journal of Engineering (JOE), Vol. 1, No. 1.," 2012.

[5] Y. S. Cho, Kim J., Yang, W.Y. and Kang C. G.,, " "MIMO-OFDM wireless communications with MATLAB”, John Wiley \& Sons, Asia, IEEE Press, pp.153-161.," 2010.

[6] F. N. X. Li, and T. Han,, "Carrier frequency offset mitigation in asynchronous cooperative OFDM transmissions,"" IEEE J. Sel. Areas Commun., vol. 56, pp. 675-685, , 2008.

[7] J. K. Yong Soo Cho, Won Young Yang, Chung-Gu Kang, "MIMO-OFDM Wireless Communication with MATLAB, ," John Wiley \&Sons (Asia), Singapore, , 2010.

[8] S. N. W. A. M. Abbas Hasan Kattoush, "'The performance of multiwavelets based OFDM system under different channel conditions," Digital Signal Processing, vol. 20, pp. 472-482, ," 2010.

[9] M. A. M. Saeed Mohseni, " "Study of the estimation techniques for the Carrier Frequency Offset (CFO) in OFDM systems" IJCSNS International Journal of Computer Science and Network Security,Vol.12, issue No.6, June 2012, pp73-80.," 2012.

[10] M. a. W. Hsieh, C., "Channel estimation for OFDM systems based on comb-type pilot arrangement in frequency selective fading channels. IEEE Trans. Consumer Electron., 44(1), 217-228.," 1998.

[11] Y. Shen, \& Martinez, E. F., , "“Channel estimation in OFDM systems” Free scale Semiconductor, Inc. ," 2006.

[12] M. Tripathi, "Effect of the Carrier Frequency Offset (CFO) on the Wireless OFDM Communications," International Journal of Emerging Technology and Advanced Engineering, 2014.

[13] R. I.-R. M. International Telecommunication Union, "“Guidelines for Evaluation of Radio", Transmission Technologies for IMT-2000.," 2007.

[14] Paul H. Moose, "“ A Technique for Orthogonal Frequency Division Multiplexing Frequency Offset Correction,”, IEEE Trans. Comm.,, vol.42, no. 10, pp. 2908-2914.." 1994.

[15] F. a. M. Classen, H., " Frequency synchronization algorithm for OFDM systems suitable for communication over frequency selective fading channels. ," IEEE VTC'94, pp. 1655-1659., 1994. 